\title{
Percepción ambiental de los productores del Ejido López Mateos, Municipio de Sayula de
}

\section{Alemán}

Environmental perception of the producers of Ejido López Mateos, Municipality of Sayula de Alemán

Martínez Martínez Marina, Carlin Bautista Isabel, Castillo Capitán Guadalupe, González Rodríguez

Eduwiges y Alvarado Gómez Luis Carlos

Universidad Veracruzana. Facultad de Ingeniería en Sistemas de Producción Agropecuarias, Región Coatzacoalcos, Campus Acayucan

${ }^{\square}$ Autor para correspondencia: $\underline{\text { marimartinez@uv.mx }}$

Recibido: $21 / 09 / 2020$

Aceptado: 15/10/2020

\section{RESUMEN}

En el presenta trabajo se muestran los resultados del estudio sobre los problemas ambientales generados por el uso excesivo de plaguicidas en la actividad agrícola, lo que trae como consecuencia la generación de envases vacíos. Estos envases requieren de un manejo correcto, un destino final preciso y controlado porque son una fuente de contaminación o riesgo tóxico para el ser humano y el medio ambiente.

La generación de envases de agroquímicos trae consigo ciertos riesgos, pero pueden ser casi nulos mediante una adecuada gestión de los envases en desuso; aunque la realidad indica que la mayor parte de los productores realiza un inadecuado manejo, ocasionado por ausencia de normas ambientales, falta de políticas públicas sobre este tipo de basura toxica y falta de información sobre cómo realizar el manejo correctamente, así como de las consecuencias perjudiciales que provoca en la salud humana y el medio ambiente.

Los principales problemas que se observan en las regiones agrícolas en la actualidad es el abandono de los envases vacíos de plaguicidas en los campos donde cultivan. Algunas prácticas comunes son las siguientes: enterrarlos, quemarlos o reutilizarlos. Sin embargo ninguno de estos métodos son acordes con el cuidado del medio ambiente ni responden a los protocolos internacionales de producción que resguardan la calidad, inocuidad de los alimentos y la salud del trabajador.

Para atender este problema se realizó un estudio los productores de la comunidad de Adolfo López Mateos, perteneciente al municipio de Sayula de Alemán. A través de una encuesta aplicada a productores del sector agropecuario, se identificaron las prácticas que realizan con los envases vacíos. Los resultados fueron que tiran los envases vacíos a la superficie donde cultivan, porque desconocen qué hacer con ellos. Sin embargo, están dispuestos a capacitarse sobre la técnica de triple lavado. Los resultados permitieron conocer las formas de percepción y cuidados del medio ambiente.

Finalmente se propuso la técnica de triple lavado como una alternativa para mejorar el cuidado del entorno, de tal manera que se pueda contribuir a reducir el consumo de plaguicidas y en su caso de envases vacíos. Así como aprender sobre el uso que se le puede dar a los envases vacíos de plaguicidas, ya que actualmente son unos de los principales problemas en el campo. 
Palabras clave: agroquímicos, basura, envases educación ambiental

\begin{abstract}
The present work shows the results of the study on environmental problems generated by the excessive use of pesticides in agricultural activity, which results in the generation of empty containers. These containers require correct handling, a precise and controlled final destination because they are a source of contamination or toxic risk for humans and the environment.

The generation of agrochemical containers brings with it certain risks, but they can be almost nil through proper management of disused containers; Although the reality indicates that most of the producers carry out inadequate management, caused by the absence of environmental regulations, lack of public policies on this type of toxic waste and lack of information on how to handle it correctly, as well as the harmful consequences it causes in human health and the environment.

The main problems observed in agricultural regions today is the abandonment of empty pesticide containers in the fields where they grow. Some common practices are as follows: bury them, burn them or reuse them. However, none of these methods are consistent with caring for the environment, nor do they respond to international production protocols that safeguard the quality, safety of food and the health of the worker.

To address this problem, a study was carried out on the producers of the community of Adolfo López Mateos, belonging to the municipality of Sayula de Alemán. Through a survey applied to producers in the agricultural sector, the practices they carry out with empty containers were identified. The results were that they throw the empty containers on the surface where they grow, because they do not know what to do with them. However, they are willing to train on the triple wash technique. The results allowed to know the ways of perception and care of the environment.

Finally, the triple washing technique was proposed as an alternative to improve the care of the environment, in such a way that it can contribute to reduce the consumption of pesticides and, where appropriate, empty containers. As well as learning about the use that can be given to empty pesticide containers, since they are currently one of the main problems in the field.
\end{abstract}

Keywords: agrochemicals, garbage, packaging environmental education.

\section{INTRODUCCIÓN}

El uso de plaguicidas se ha hecho casi indispensable en la agricultura moderna a pesar de que actualmente en países desarrollados se ha comenzado a disminuir el uso de estos productos, pero en países en vías de desarrollo se siguen aplicando de manera intensiva.
Durante años, se ha observado que los envases de agroquímicos son tirados de manera irresponsable en canales de riego, arroyos, zanjas, brechas, barrancas, en el campo abierto y en algunos casos son quemados, enterrados o reciclados para almacenar agua o alimentos. Todas estas prácticas generan focos de contaminación al ambiente (aire, tierra, cuerpos 
de agua) y en ocasiones problemas de intoxicación en las personas.

El excesivo empleo plaguicidas en la agricultura provoca daños a la salud y al medio ambiente, sobre todo a la población que labora en el sector agrario, quienes tienen el mayor contacto con estos productos por ser uno de los principales insumos para la producción agrícola.

El manejo inapropiado de plaguicidas ha provocado daños en la salud de los usuarios directos, y a la población que está expuesta indirectamente así como al ambiente. Ante esto se ha planteado la urgente necesidad de formular medidas preventivas que garanticen el manejo seguro de estas sustancias, entre ellas se encuentran las siguientes: la protección física de los usuarios, la reducción de la cantidad de producto usado en los cultivos, la minimización de daños al ambiente (especialmente cuerpos de agua), el acopio y la disposición de envases, residuos y producto caduco son algunas de las medidas que se consideran prioritarias a nivel mundial.

Por otro lado, podemos señalar, la falta de capacitación a trabajadores agrícolas y productores quienes directamente hacen las aplicaciones de plaguicidas, además de la falta de equipo de protección apropiado ocasiona que se incrementen los riesgos de intoxicación. INTAGRI (2017).

En México en el año 2012, según reportes de los Comités Estatales de Sanidad Vegetal, se generan 50 millones de envases de agroquímicos, lo cual equivale a 6,020 toneladas. Los envases de plástico, ya sean rígidos o flexibles representan más del $80 \%$ de estos, dejando muy por debajo a los envases de papel y metal. (INTAGRI, 2017).
En los últimos años debido a la creación y firma de tratados internacionales en los que diversos países forman parte, los planes de manejo para los envases vacíos de agroquímicos se han establecido a nivel mundial. En Australia y Canadá, se han logrado implementar programas de recolección y recuperación de envases. En Alemania, se recolectan los envases triplemente lavados y se incineran en hornos siderúrgicos o de cemento, o bien se reutilizan para fabricar materiales para la construcción, operación que a la fecha se lleva a cabo con éxito.

En otros países existe la posibilidad de colocar los envases en rellenos sanitarios, ya sean municipales o particulares; pero siempre bajo control, sin embargo depende de las leyes de cada país. En México, al día de hoy, tal práctica no está permitida.

Es importante que los productores tomen en cuenta que el uso de plaguicidas implica un riesgo alto para la salud. La población afectada principalmente es la que trabaja en la fabricación y manipulación de productos, además de la comunidad en general que maneja estas sustancias a nivel del hogar y en actividades secundarias, como lo informa la asociación civil Campo Limpio.

El envase vacío de un plaguicida es un producto peligroso, en la mayoría de casos no se le hace una disposición adecuada, generando así, grandes problemas como: reutilización de envases en actividades para consumo humano como para animal, mala disposición en suelos y cuerpos de agua, enterramiento, quema incontrolada y piratería; esta última, permite que los envases que no fueron gestionados de la mejor manera; sean reutilizados para envasar otro tipo de producto que no cuente con las propiedades idénticas a la etiqueta original. 
Según Avendaño (2015), el desconocimiento de la comunidad es uno de los principales problemas en el manejo de los planes de gestión pos consumo, puesto que en la mayoría de los casos, olvidan hacer conciencia de la importancia de aplicar dichos planes de manejo de envases, el horario de recolección y la ubicación de los centros de acopio para la recepción de envases.

\section{MATERIALES Y MÉTODOS}

El Municipio de Sayula de Alemán es uno de los 212 municipios en que se encuentra dividido el estado mexicano de Veracruz de Ignacio de la Llave, ubicado en la Región Olmeca y su cabecera es el pueblo de Sayula de Alemán.

Adolfo López Mateos se ubica en el estado de Veracruz de Ignacio de la Llave. En el Municipio de Sayula de Alemán. Localizado en una altura de 41 metros. La población total es de 255 habitantes de cuales 129 son hombres o niños y 126 mujeres o niñas. La latitud (decimal) es 17.633889 y la longitud en el sistema decimal es $-94.980833$.
En el ejido Adolfo López Mateos, perteneciente al Municipio de Sayula de Alemán, se realizó una investigación de campo de carácter exploratoria con la finalidad de conocer el tipo de envase que se generan por consumo de productos químicos en la agricultura y ganadería.

Además se exploró sobre el conocimiento que tienen los productores, sobre el cuidado del medio ambiente. Se empleó como herramienta de investigación un cuestionario de 20 preguntas que se aplicó los agricultores de la localidad.

Para llevar a cabo la aplicación del instrumento se realizaron visitas domiciliarias a los productores para conocer el tipo de insumos que emplean en sus cultivos.

\section{RESULTADOS Y DISCUSIÓN}

Resultados del estudio realizado en el ejido Adolfo López Mateos, municipio de Sayula de Alemán, Veracruz. En él se muestran las diferentes variables estudiadas con sus respectivas gráficas.

\section{NIVEL DE ESTUDIOS}
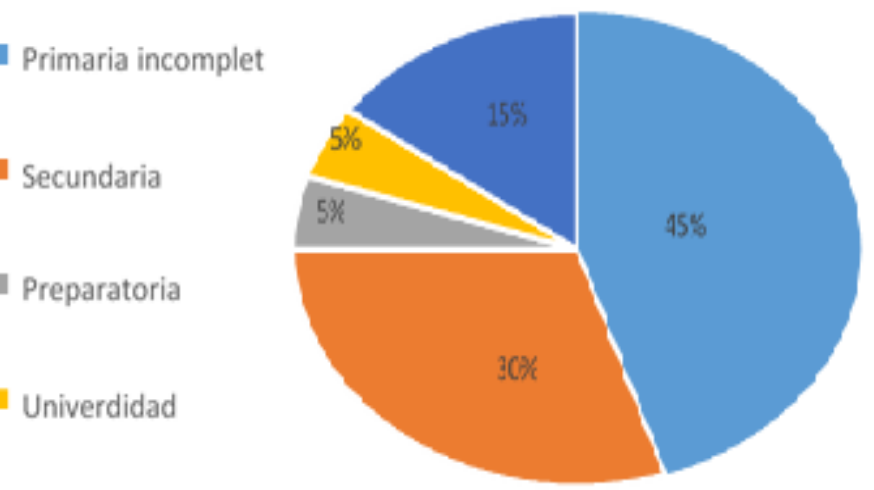

Figura 1. Nivel de estudios en personas encuestadas en el Ejido Adolfo López Mateos, municipio de Sayula de Alemán, Veracruz. 
De acuerdo con los encuestados el $15 \%$ no tiene ningún nivel de escolaridad, el $45 \%$ tiene primaria incompleta, el 5\% son bachilleres, tan solo el $5 \%$ estudiaron la universidad.

A la pregunta sobre la actividad productiva, encontramos que la mayor parte de la comunidad
(50\%) se dedica a la agricultura y siembran diferentes tipos de cultivos, pero los que más predominan son el maíz y frijol. El $25 \%$ se dedica a la ganadería y el otro $25 \%$ a ambos. Esto indica que en esa comunidad existe más persona que se dedican a cultivar alimentos básicos. Lo cual ocasionamos más generación de envases vacíos de agroquímicos.

\section{¿Actividad productiva a la que se dedica?}

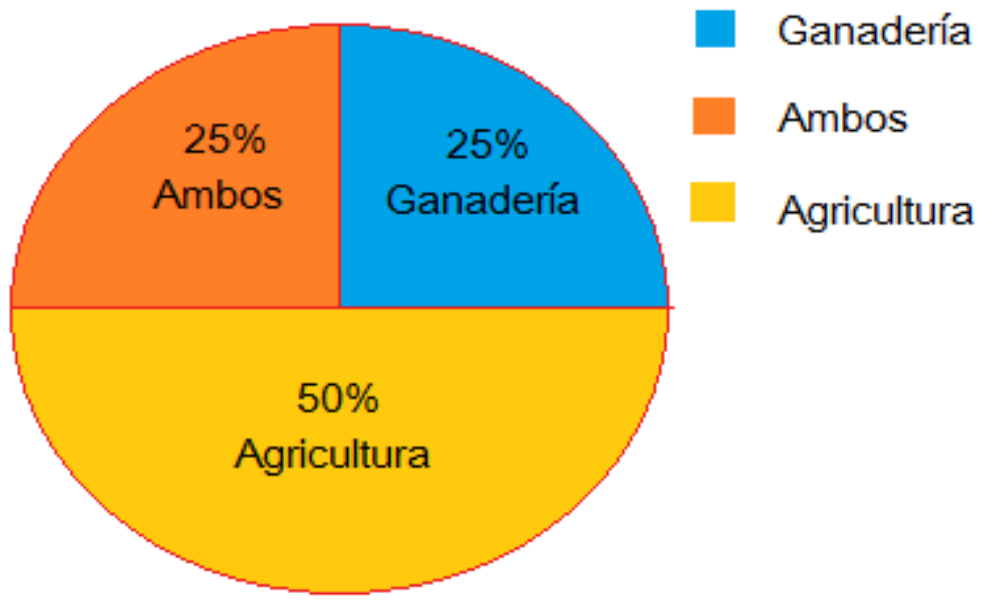

Figura 2. Actividad productiva de las personas encuestadas en el Ejido Adolfo López Mateos, municipio de Sayula de Alemán, Veracruz.

El cultivo que predomina en esta comunidad es el cultivo del maíz ya que cuenta con el $43 \%$ de siembra, el frijol es el segundo cultivo más sembrado con el $25 \%$, el $10 \%$ siembra calabaza y el $8 \%$, al igual que la calabaza el $8 \%$ cultiva el pipián. 


\section{¿Usted que cultivos siembra?}

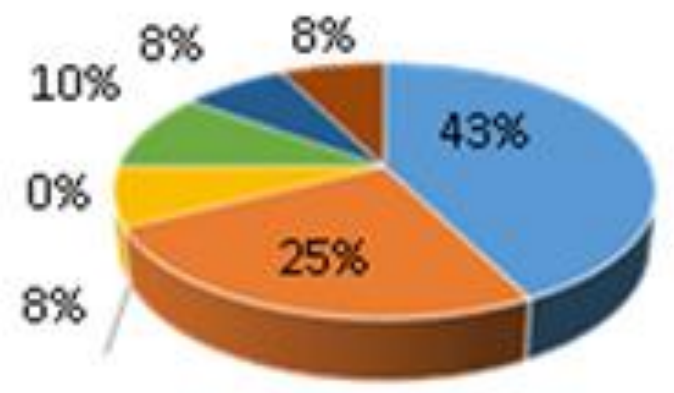

\section{Maiz $=$ Frijol $\mid$ Caña $=$ Pasto \\ Café =Calabaza" Yuca =Pipian}

Figura 3. Principales cultivos que siembran las personas encuestadas en el Ejido Adolfo López Mateos, municipio de Sayula de Alemán, Veracruz.

¿Qué hace con los envases vacíos de siembran, el 5\% los tiran en el carro de basura, agroquímicos?

el 15\% los reutilizan para almacenar agua, y el

De acuerdo con los entrevistados el $45 \%$ señaló $35 \%$ los queman.

que tiran las botellas a la superficie donde los

\section{¿QUÉ HACE CONLOS ENVASES VACÍOS DE AGROQUÍMICOS?}

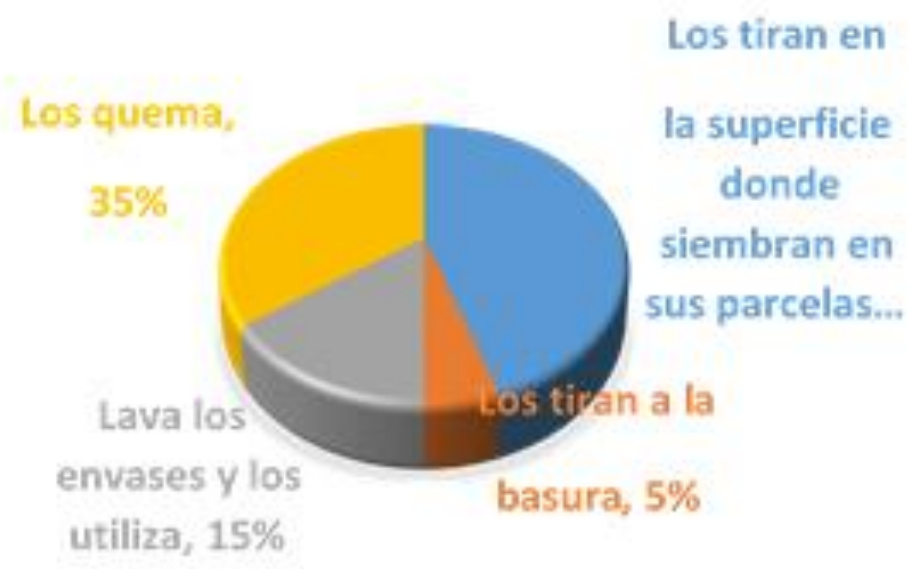

Figura 4. Destino de los envases vacíos de agroquímicos que utilizan las personas encuestadas en el Ejido Adolfo López Mateos, municipio de Sayula de Alemán, Veracruz. 
Conocimiento sobre la técnica del triple lavado. De acuerdo con los entrevistados solo el $15 \%$ conoce en que consiste la técnica del triple lavado, y el $85 \%$ desconoce completamente en que consiste la técnica.

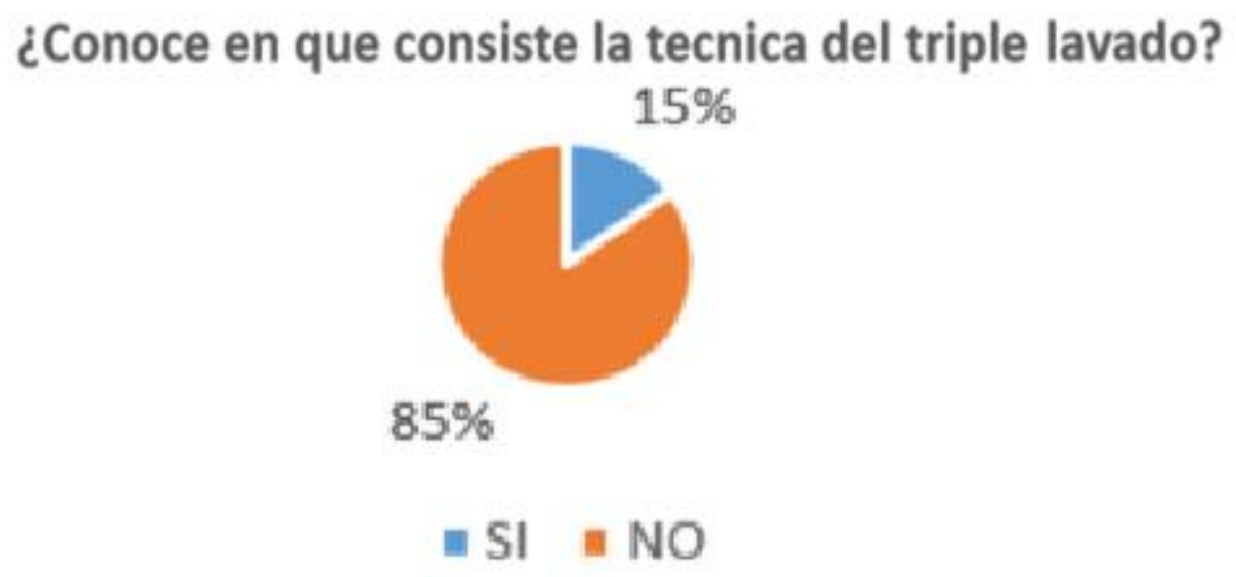

Figura 5. Porcentaje de personas que tienen conocimiento de la técnica del triple lavado según la encuesta realizada en el Ejido Adolfo López Mateos, municipio de Sayula de Alemán, Veracruz.

A la pregunta sobre si esta de acuerdo o no con la contaminación ambiental, los entrevistados $90 \%$ señaló que nó, mientras que el $10 \%$ esta de acuerdo con la contaminación ambiental. Este resultado nos permite identificar que la población esta conciente de que enfrentamos un problemande contaminación, por ello no esta de acuerdo. Esta respuesta nos habre la posibilidad de emprender proyectos de educación ambiental.

\section{¿Usted está de acuerdo con la contaminación ambiental?}

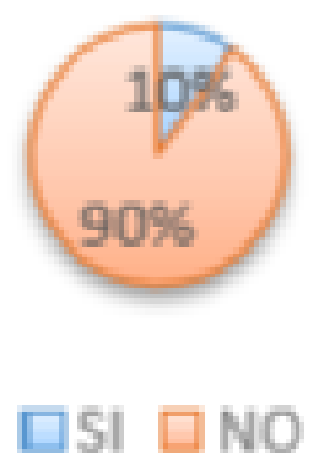

Figura 6. Porcentaje de personas que están de acuerdo con la contaminación ambiental según la encuesta realizada en el Ejido Adolfo López Mateos, municipio de Sayula de Alemán, Veracruz están de acuerdo con la contaminación ambiental. 
Al preguntar su opinión sobre tirar la basura al suelo encontramos que el $100 \%$ de la población entrevistada dijo que no es bueno. Esto indica que la población entrevista es consciente del cuidado de la basura.

\section{¿Cree que arrojar basura al piso es bueno?}

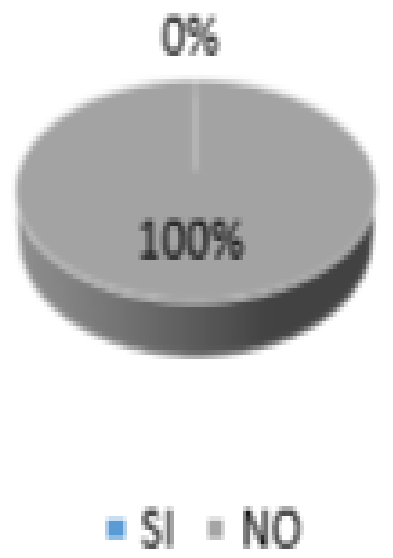

Figura 7. Porcentaje de personas que creen que arrojar basura al suelo es una práctica que consideran como buena las personas encuestadas en el Ejido Adolfo López Mateos, municipio de Sayula de Alemán, Veracruz. 


\section{DISCUSIÓN}

Al realizar este trabajo me pude percatar que existen muchos problemas ambientales que nosotros mismo como seres humanos generamos sin tomar en cuenta las consecuencias que estamos ocasionando al ambiente. Hoy en día los plaguicidas unos de los principales contaminantes en la agricultura, a causa del exceso en el uso de agroquímicos sintéticos. Y que no solo se utiliza en el la agricultura, sino también en los hogares y en la ganadería.

\section{CONCLUSIÓN}

En la comunidad de Adolfo López Mateos existe buena disposición de la población para participar en programas de educación ambiental y cuidado del medio ambiente. Por otro lado, hace falta promover acciones de educación ambiental y manejo de envases post consumo de productos agroquímicos.

Se concluye que es importante crear alternativas de apoyo a los agricultores a través de estrategias de educación ambiental que nos permitan reducir los efectos de la contaminación. Además hace falta promover talleres de buenas prácticas sobre el empleo de agroquímicos y sus medidas de seguridad en la aplicación. También se identifica como importante promover acciones de responsabilidad en la población para reducir el consumo y generación de envases.

\section{LITERATURA CITADA}

Alejandro, R. C. (2014). Diseño De Un Plan De Manejo De Envases Vacíos De Productos Fitosanitarios En La Asociación Agropecuaria Quinlata En El Cantón Patate De La Provincia De Tungurahua. Tungurahua: Universidad Tecnológica Equinoccial.
Bedmar, F. (2011). Informe especial sobre plaguicidas agricolas . Facultad de Ciencias Agrarias Universidad Nacional de Mar del Plata, 2-8.

Carola, L. (2011). Pesticides. Chapter 42, http://eknygos.lsmuni.lt/springer/99/801809.pdf

Clasificasiones mas importantes de los plagicidas (s.f.). Obtenido de Gobierno de Mexico:

https://www.gob.mx/cms/uploads/attach ment/file/25247/clasificaciones.pdf

Daniela, P. V. (01 de agosto de 2018). Causas y consecuencias de las problematicas actuales en la gestion de envases plaguicidas de uso agricola en cundinamarca. Obtenido de fundacion universidad de america: https://repository.uamerica.edu.co/bitstrea $\mathrm{m} / 20.500 .11839 / 6919 / 1 / 5121873-2018$ IIGA.pdf

Gabriel Bertoldo Castillo Reséndiz, C. E. (2012). Plan de manejo y recolección de envases vacios de plaguicida. Obtenido de conservemos un campo limpio": http://www.cesaveq.org.mx/cesa3/page/d ctos/inocuidad/plamrevp.pdf

INTAGRI. (2017). Técnica del Triple Lavado de Envases de Plaguicidas. Serie Fitosanidad. Núm. 76. Artículos Técnicos de INTAGRI. México, págs. https://www.intagri.com/articulos/fitosani dad/tecnica-del-triple-lavado-de-envasesdeplaguicidas

José, M. J. (1980). Pesticidas: clasificación y riesgos principales. Instituto nacional de seguridad e higine en el trabajo , 1-4. 
Ministerio de Ambiente, V. y. (2003). Guías para la Gestión Ambiental Responsable de los Plaguicidas Químicos de Uso Agrícola en Colombia. colombia : Segunda edicion Obtenido de http://cep.unep.org/repcar/capacitacion-yconcienciacion/andi/publicacionesandi/G uia\%20ambiental\%20plaguicidas.pdf

OMS, F. (Mayo de 2008). Código internacional de conducta sobre la distribución y utilización de plaguicidas . Italia: Oficina de Intercambio de Conocimientos, Investigación y Extensión, FAO,

OMS, O. M. (2010). Clasificación OMS de los plaguicidas. Obtenido de Programa Internacional de Seguridad de las Sustancias Químicas: https://www.who.int/ipcs/assessment/publ ic health/pesticides/es/

SEMARNAT, S. d. (s.f.). Situación Actual de los Envases Vacíos de Plaguicidas. Obtenido de Direccion general de gestion integral de materiales $y$ actividades riesgosas

SERMARNAT. (Febrero de 2018). Elementos para desarrollar una estrategia integral para la gestión responsable de plaguicidas en méxico. Obtenido de Disponible en: https://www.gob.mx/cms/uploads/attach ment/file/451603/Elementos_para_Desarr ollar_una_Estrategia_Integral_de_Ma

Tamayo, D. S., \& M, D. A. (2014). Efectos de los plaguicidas sobre el ambiente y la salud. Revista Cubana de Higiene y Epidemiología vol.52, 1-6.

Villalba, D. P. (01 de AGOSTO de 2018). Causas y consecuencias de las problemáticas actuales en la gestión de envases plaguicidas de uso agrícola en cundinamarca. Fundación universidad de america, págs. 19-43. Virya, E. d., \& Gustavo Herrera, F. R. (2013). Uso de plaguicidas en cultivos agrícolas como herramienta para el monitoreo de peligros en salud. Uniciencia , 4-27.

Viviana, M. P. (01 de octubre de 2014). Identificación de alternativas para la disposición final de los envases de plaguicidas de uso agricola. Corporación Universitaria Lasallista, págs. 31-66. 
Martínez et al., 2020

Copyright (c) 2020 Marina Martínez Martínez, Isabel Carlin Bautista, Guadalupe Castillo Capitán, Eduwiges González Rodríguez y Luis Carlos Alvarado Gómez

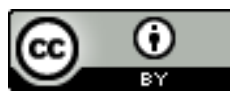

Este texto está protegido por una licencia Creative Commons $\underline{4.0 .}$

Usted es libre para Compartir — copiar y redistribuir el material en cualquier medio o formato — y Adaptar el documento —remezclar, transformar y crear a partir del material - para cualquier propósito, , incluso para fin es comerciales, siempre que cumpla la condición de:

Atribución: Usted debe dar crédito a la obra original de manera adecuada, proporcionar un enlace a la licencia, e indicar si se han realizado cambios. Puede hacerlo en cualquier forma razonable, pero no de forma tal que sugiera que tiene el apoyo del licenciante o lo recibe por el uso que hace de la obra.

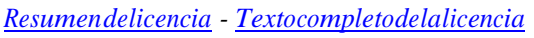

